\title{
Technology in dementia care
}

\author{
Suzanne Cahill ${ }^{\mathrm{a}, *}$, Jurate Macijauskiene ${ }^{\mathrm{b}}$, Aase-Marit Nygård ${ }^{\mathrm{c}}$, Jon-Paul Faulkner ${ }^{\mathrm{a}}$ and Inger Hagen $^{\mathrm{d}}$ \\ ${ }^{a}$ Dementia Services Information and Development Centre, St. James Hospital, Dublin, Ireland \\ ${ }^{\mathrm{b}}$ Geriatric Clinic, Kaunas University of Medicine, 3000 Kaunas, Lithuania \\ ${ }^{\mathrm{c}}$ Norwegian Centre for Dementia Research, Ullevål University Hospital, 0407 Oslo, Norway \\ dP.O. Box 7167 Majorstua, 0307 Oslo, Norway
}

\begin{abstract}
The aim of this paper is to provide a clinical overview of Alzheimer's disease and the related dementias and to detail the progressive losses - physical, social and psychological - experienced by the individual diagnosed and the implications such losses have for user requirements in assistive technologies. The paper argues that in the absence of a cure for Alzheimer's disease and the related dementias, more innovative social care policies will need to be developed, designed to address the unique and complex needs of all those diagnosed. Although, assistive technologies have much potential in helping to compensate for the multiple losses experienced by those diagnosed it is argued that their potential has not been fully realised. The paper opens with a discussion of when Alzheimer's disease was first clinically identified, and identifies reasons behind the present increase in numbers of people presenting with this disability across the world. A brief description of the clinical symptoms of the most common dementias is provided and of how the disease can adversely affect the daily living of people affected. Examples are given on how technology can support people with dementia and their caregivers and what requirements should be considered regarding their function and design.
\end{abstract}

Keywords: Dementia, demography, technology, user requirements

\section{Background, definitions and demography}

Dementia is now recognized as a global issue of increasing importance, affecting some 24 million people around the world [9]. The disability weight for dementia is high [30], the cost of care is enormous [16] and despite a burgeoning body of medical, neuropsychological and clinical research, there is still no cure for Alzheimer's disease and the related dementias and much uncertainty exists surrounding its exact cause [24]. Accordingly in the absence of a cure or effective treatments, Alzheimer's disease and the related dementias have very significant implications for health and social care policies.

Medically, the term dementia refers to the global impairment of higher cortical function, including memory, the capacity to solve the problems of daily living,

\footnotetext{
*Address for correspondence: Suzanne Cahill, Dementia Services Information and Development Centre, St. James Hospital, Dublin 8, Ireland. E-mail: scahill@stjames.ie.
}

the performance of learned perceptuo-motor skills, the correct use of social skill and the control of emotional reactions, in the absence of gross clouding of consciousness [13]. Up until relatively recently, this (bio-) medical definition of the disease has tended to underpin and dominate the policy, practice and research agenda [6]. More recently however, with the pioneering work of Tom Kitwood and other social and behavioural scientists, a more holistic approach to defining dementia has emerged, with due recognition being given to the critical role played by social, psychological and environmental factors in illness presentation and in care management. This has led to a new wave of interest being invested into better understanding the experiences of people with dementia and the evolution of a corresponding new culture of dementia care [15].

\subsection{The historical emergence of Alzheimer's disease}

Alzheimer's disease was first identified one hundred years ago (1906) by a Bavarian clinical psychiatrist, 
Alois Alzheimer, who in describing the symptoms of a 50 year old patient, referred to her memory loss problems, disorientation and hallucinated depression. $\mathrm{Cu}-$ riously, in attempting to diagnose this woman's condition, Alzheimer argued, that a new disease entity had been discovered, which in his view, was conceptually different from pre-senility or dementia praecox. Accordingly, from its early identification through its distinctive plaques and tangles, and up until the mid 70's, Alzheimer's disease was considered a pre-senile dementia with symptoms manifesting in those aged between 40 and 60 . In contrast senile dementia or senility was considered a normal part of ageing, its symptoms occurring in those over the age of 60 .

\subsection{The re-classification of Alzheimer's disease}

An important breakthrough in the evolution of Alzheimer's disease occurred in the 1960's when Katzman [14], an American physician on examining brain biopsies, discovered that many of the brains of patients with suspected hydrocephalus had the same plaques and tangles characteristic of Alzheimer's disease and concluded that clear similarities existed between presenile dementia of the Alzheimer's type and senile dementia. His work was revolutionary in that it called for the elimination of age as a criterion for Alzheimer's disease. Most importantly his work challenged the contemporary view that senility was a normal part of ageing: growing old was no longer seen to cause senility but rather the latter was now seen as a medical condition with pathological symptoms and characteristics. Thus began the gradual erosion of the distinction between normalcy and pathology, pre-senile and senile dementia, and the emergence of much larger numbers of people presenting with Alzheimer's disease and dementia. Led by the United States, this work set the stage for the commencement of a large dementia movement, a phenomenon which has fully taken place across the world.

\subsection{The epidemic of dementia: A myth or reality?}

A second and more contemporary factor explaining the significant growth in numbers of people presenting with Alzheimer's disease around the world today has been population ageing. The world's population is ageing at an unprecedented rate [18]. Over the next 40 years for example, the number of older people across the world is expected to increase from 6.3 billion today to 8.9 billion in 2050 [25]. Population ageing is of particular relevance to Alzheimer's disease and the related dementias since we know that dementia prevalence rates rise sharply with age, doubling every 5 years after the age of 65 from $3 \%$ at age 70 to $20 \%$ at 85 years [26]. Accordingly in the absence of a cure, population ageing along with the expected growth in numbers of people with dementia will mean very significant challenges to governments, policy makers, caregivers and researchers. Clearly a multi-disciplinary approach, drawing together, doctors, nurses, occupational therapists, social scientists, engineers, architects, and technicians will be needed to tackle the magnitude of the challenge.

Detailed estimates of the global prevalence of dementia recently generated by a world expert group (ADI) have shown that across the world, 4.6 million new cases of dementia will emerge every year and that the numbers of people living with dementia will almost double every 20 years from 42.3 million in 2020, to 81.1 million in 2040 [9]. More detailed analysis shows that in 2001, those countries with the largest number of people with dementia were China ( 5 million), the EU (5 million) the US (2.9 million) India (1.5 million), Japan (1.1 million), Russia (1.1million) and Indonesia (1.0 million). Given however the disproportionately larger numbers of older people likely to be living in developing countries in future years, their estimates suggest that in the future the numbers of people with dementia in the developing world will vastly exceed those in the developed world. The largest growth in numbers of people with dementia will occur in India, China, South Asia and the Western Pacific where a growth of 314$336 \%$ will occur. The second largest growth will occur in Latin American countries.

The challenge that dementia will pose therefore needs to be understood against this background of world demographic trends, increased life expectancy, changes in the classification of Alzheimer's disease, and the particular difficulties that low income countries will in the future experience given the high numbers of people likely to experience dementia there. Bearing in mind the projections described in recent international works $[11,26,28]$, evidence now exists on which to develop more responsive dementia services across the world [9]. Since most care will continue to take place in the community and since there will be a paucity of women available to provide traditional informal care carefully designed technological interventions will need to become an integral component of home care packages and a key priority in planning community services. 


\section{Clinical symptoms of some dementia diseases}

Dementia is a syndrome characterized by the disturbance of multiple higher cortical functions, including memory, thinking, orientation, comprehension, calculation, learning capacity, language and judgement. Impairments of cognitive function are commonly accompanied by deterioration in emotional control, social behaviour, or motivation [27]. Alzheimer's disease is by far the most common type of dementia (accounting for more than $65 \%$ of all cases) followed by vascular dementia (approx. 25\%). Rarer dementia disorders include dementia with Lewy bodies and fronto-temporal dementia. Different types of dementia and different stages of development bring about different symptoms in psychological, behavioural and motoric functioning. Vascular dementia is a heterogonous group and the clinical deficits depend on the size, localization and type of cerebral damage. The deterioration of clinical symptoms, cognitive functions and behavioural problems in vascular dementia is stepwise and fluctuating [8]. Dementia with Lewy bodies is characterised by fluctuating cognition with variations in attention and alertness, visual hallucinations, and motor features of Parkinsonism [22]. Typical symptoms of fronto-temporal dementia include challenging behaviour and relatively good memory in the early phase. However, some changes are fairly common in the development of all dementias such as memory loss, agnosia- the inability to recognize people or objects even when basic sensory modalities, such as vision, are intact and apraxia or loss of ability to execute or carry out skilled movements and gestures, despite having the desire and the physical ability to perform them.

\subsection{Alzheimer's disease}

Alzheimer's disease is characterised by an insidious onset and by progressive impairment of memory and other cognitive functions [23]. Memory loss, especially of short-term memory, is an early sign. Disorientation in time and place, misplacing things (as placing things in unusual places), are often experienced in dementia and may become frustrating not only for persons with dementia, but also for their caregivers. Problems with language manifest as difficulties occur finding the right words, substituting usual words with neologisms and rustic speech. Difficulties in performing familiar tasks, (which for most people require no thinking) may occur, and activities of daily living may become impaired, and may make people with dementia dependent on others. Due to poor judgement persons with dementia may dress inappropriately, behave in an improper manner, and neglect household chores. Loss of initiative may make the person with dementia very passive, losing interests in leisure activities. Typical changes in personality include apathy or agitation, irritability and suspiciousness. At the mild stage of Alzheimer's disease, people may be able to live almost independently, although some may need their homes rearranged and more formal or informal support. With the progression of the illness, many persons with dementia lose their autonomy and need close supervision and support services. In severe stages, people with dementia need support in very basic daily activities (eating, hygiene etc.), and caregiving can be very strenuous, although emotional contact may remain intact.

\subsection{Challenging behaviour}

The neuropsychiatric changes in dementia are nearly universal and may result in extremely challenging management problems [17]. Broadly speaking these are often classified into psychological and behavioural symptoms. Psychological symptoms include - delusions, hallucinations, depression, anxiety, and misidentifications. Behavioural symptoms may include - aggression, wandering, sleep disturbances, inappropriate behaviour [19]. Irrespective of the dementia subtype in most cases, people with dementia will, during the course of the illness, present with some of these behavioural and psychological symptoms. Only minor differences in the prevalence of behavioural disturbances in Alzheimer's disease and vascular dementia have been noted [10]. A general rule of thumb with regard to these challenging behaviors is that drug interventions should only ever be used after appropriate non-drug approaches have first been tried or where drugs are considered essential to avoid harm to self or others.

\section{The disability of dementia: How can technology assist?}

As mentioned, people with dementia experience progressive cognitive impairments that typically commence with short term memory problems but can encompass language deficits, difficulties initiating tasks, planning, monitoring and regulating behaviour, and visuospatial difficulties, agnosia (loss of ability to recognize familiar objects or people and apraxia (loss of abil- 
ity to carry out complex purposive movements). Whilst drugs have for some time been used and approved by health organizations for the treatment of mild to moderate Alzheimer's disease, these drugs do not cure, reverse or tackle the underlying root problem causing the dementia. Therefore in the absence of a cure, more innnovative approaches need to be developed to help promote independence and maximise quality of life.

In this context, assistive technologies offer much potential and can make a very significant difference to the lives of people with dementia and to their primary caregivers. Indeed it has been noted that technologies should be part of a home package and should be provided in a thoughtful, sensitive ethical way [21]. Technologies can assist people to maintain their independence improve quality of life. Recent initiatives including the Astrid project [20] ENABLE (findings from which are described in this issue and in special issue of Dementia [7]) and the Safe at Home project [29] demonstrate the opportunities such technology can create for people with a dementia. The overall opportunities technology can create for people with dementia however have to date not been fully maximised [20].

\subsection{User requirements}

Some general principles of user requirements were described by Bjørneby [3]. The technologies should:

- give a feeling of independence to the person;

- support the person in making choices;

- have a positive impact on his/her life;

- support skills maintained or do not emphasise lost skills;

- not focus on the user as a person with disabilities, but supports the self image of being a person with abilities;

- remind the solutions that existed before;

- The use of the products is possible by the information visible/available at all times.

Bjørneby et al. [3,4] further elaborated on this and presented an adaptation of Design for All principles for development of assistive technology for people with dementia. For each of the 7 Design for All principles, the needs of people with dementia and the respective requirements are presented.

In addition to the general principles referred above product-specific user requirements need to be defined as part of the development process; how should the general requirements be set out in practice during development of a specific technological device or system?
In this connection, it is also important to keep in mind what need(s) the technology aims to meet, what abilities would be required in order to make use of it (e.g. read, hear a sound, attention) and the risk of unwanted effects, such as confusion by (suddenly) hearing a voice message.

Furthermore, the role of the user in handling the technology is important for design, function as well as ethical questions to be addressed in connection with the implementation and use of the technology. The technologies can be categorised as follows:

- Devices that are operated by the person (radio, TV, (mobile) telephone, car);

- Systems and devices that others have installed and maintain, but which the person uses (electricity, water supply system, air condition);

- Monitoring and surveillance systems and devices which are either:

* activated by the user (safety alarms);

* activated automatically when an incident occurs (fire alarm, fall alarm);

* monitors continuously or when the operator decides (cameras installed at public places, tagging devices).

Obviously, when the person with dementia has an active role in handling the assistive technology, due considerations to the clinical symptoms must be taken.

\subsection{User needs}

Choosing the appropriate assistive technology is not always easy and there is a wide range of different technologies that can be adapted and used for people with dementia to help them cope with the practical problems encountered in daily living. Broadly speaking these technologies, fall into 4 main categories, namely (i) those used to promote safety (examples here might include night lamps, flood detectors, cooker switch off devices), (ii) foster communication and address memory loss problems (picture button telephones), (iii) provide multi-sensory stimulation (picture-gramaphone) and (iv) act as memory enhancers (reminder messages, electronic calendars, item locators, and medicine reminders). In an era where micro-chips are being inserted into the living brains of people affected by brain injury, the potential technologies have to address dementia-related problems such as agnosia and apraxia is enormous. Further advances in these areas for those diagnosed with dementia are likely to be on the horizon. 
It also needs to be remembered that people react differently to different assistive technologies and there are no quick fix solutions in dementia care. Nor do solutions necessarily have to be highly technical. Whilst one person might derive much pleasure from a sophisticated multi-sensory media system linked up to a computer terminal, another might prefer a simple audio-tape of their individual music preferences. The most controversial application of technology in dementia care is seen in the application of tracking and surveillance equipment [1]. Proponents of these technologies argue that the technologies reduce caregiver stress and increase individual autonomy since they allow the person with dementia freedom to move around as they wish [2]. Critics however argue that these technologies are an infringement of people's civil liberties and undermine the personhood of the individual $[5,12]$.

Finally the nature of dementia may make people cautious and suspicious of trying out new devices. User requirements should therefore take into consideration, individual and caregiver needs, the design of the product, including its familiarity and the fact that no new learning should be required on the part of the person with a dementia. A comprehensive assessment of needs should take place ideally at home with a health service professional fully trained in dementia care. Pre-testing is critical to ensure that the chosen product is reliable and effective. Open and in-depth discussion with the individual and his or her family caregiver must always be a priority. Ultimately it is important to find a product that suits the individual and is not complex or stigmatizing. There is also a higher chance of product success if it is introduced at an early stage in the course of the dementing illness so that the person can gradually become accustomed to it. Finally it needs to be remembered that technologies will never replace human individualised personal care. At best, technologies can only supplement but can never substitute for the type of human care and support we all cherish.

\section{References}

[1] A. Astell, Developing Technology for People with Dementia, Psychiatric Times Vol XXIII: Issue 13 Nov (2005) URL: http: //psychiatrictimes.com/article/showArticle.jhtml?articleId= 174402656.

[2] K.D. Bail, Electronic tagging of people with dementia. Devices may be preferable to locked doors, BMJ 326(7383) (2003), 218.

[3] S. Bjørneby, P. Duff and O. Mãki, Developing assistive technology for people with dementia, in: Assistive Technology - Shaping the Future, G. Craddock, ed., IOS Press, 2003, pp. 781-786.
[4] S. Bjørneby, P. Topo and T. Holthe, Technology, Ethics and Dementia, Norwegian Centre for Dementia Research, 1999.

[5] S. Cahill, Technologies may be enabling, BMJ 326 (2003), 281.

[6] R. Cheston and M. Bender, Understanding Dementia: The man with the Worried Eyes, Jessica Kingsley, London 1999.

[7] Dementia, The International Journal of Social Research and Practice 3 (2004), 259-350.

[8] T. Erkinjunti, Vascular cognitive impairement, in: Dementia, A. Burns, J. O'Brien, D. Ames and H. Arnold, eds, 2005, pp. 533-553.

[9] C.P. Ferri, M. Prince, C. Brayne, H. Brodaty, L. Fratiglioni, M. Ganguli, K. Hall, K. Hasegawa, H. Hendrie, Y. Huang, A. Jorm, C. Mathers, P.R. Menezes, E. Rimmer and M. Scazufca, Global prevalence of dementia: A Delphi consensus report, Lancet 366 (2005), 2112-2117.

[10] J.L. Fuh, S.J. Wang and J.L. Cummings, Neuropsychiatric Profiles in patients with Alzheimer's Disease and vascular dementia, J Neurol Neurosurg Psychiatry 76 (2005), 13371341.

[11] H.C. Hendrie, B.O. Osuntokun and K.S. Hall, Prevalence of Alzheimer's disease and dementia in two communities: Nigerian Africans and African Americans, Am J Psychiatry 152 (1995), 1485-1492.

[12] J.C. Hughes and S.L. Louwe, Electronic tagging of people with dementia who wander, BMJ 325 (2002), 847-884.

[13] A.F. Jorm, A.E. Korten and A.S. Henderson, The prevalence of dementia: a quantitative integration of the literature, Acta Psychiatr Scand 76 (1987), 465-479.

[14] R. Katzman, Normal pressure hydrocephalus contemporary, Neur 15 (1977), 69-92.

[15] T. Kitwood, Dementia Re-considered, The Person Comes First, Buckingham: Open University Press, 1997.

[16] K.M. Langa, M.E. Chernew and M.U. Kabeto, National estimates of the quantity and cost of informal caregiving for the elderly with dementia, J Gen Intern Med 16 (2001), 770-778.

[17] I. Leroi and C.G. Lyketsos, Neuropsychiatric aspects of dementia, in: Dementia, A. Burns, J. O'Brien, D. Ames and H. Arnold, eds, 2005, p. 55.

[18] P. Lloyd-Sherlock, Population ageing in developed and developing regions: implications for health policy, Soc Sci Med $\mathbf{5 1}$ (2000), 887-895.

[19] J. Luxenberg, Clinical issues in the behavioural and psychological symptoms of dementia, Int J Geriatr Psychiatry, 15 (2000), S2-S4

[20] M. Marshall, ASTRID: A Social and Technological Response to Meeting the Needs of Individuals with Dementia and their Carers: A Guide to using Technology within Dementia Care, Hawker Publications Ltd., London, UK, 2000.

[21] M. Marshall, Foreword, in: Assistive Technology in Dementia Care, J. Woolham, ed., Hawker Publications Ltd, London UK, 2006.

[22] I.G. McKeith, D. Galasko, K. Kosaka, E.K. Perry, D.W. Dickson, L.A. Hansen, D.P. Salmon, J. Lowe, S.S. Mirra, E.J. Byrne, G. Lennox, N.P. Quinn, J.A. Edwardson, P.G. Ince, C. Bergeron, A. Burns, B.L. Miller, S. Lovestone, D. Collerton, E.N. Jansen, C. Ballard, R.A. de Vos, G.K. Wilcock, K.A. Jellinger and P.H. Perry, Consensus guidelines for the clinical and pathologic diagnosis of dementia with Lewy bodies (DLB): report of the consortium on DLB international workshop, Neurology 47(5) (1996), 1113-1124.

[23] G. McKhann, D. Drachman, M. Folstein, R. Katzman, D. Price and F.M. Stadlan, Clinical diagnosis of Alzheimer's disease: report of the NINCDS-ADRDA work group under auspices 
of Department of Health and Human Services task force on Alzheimer's Disease, Neurology 34 (1984), 939-944.

[24] M. Nair, Nurse Management of the Patient with Alzheimer's Disease, British Journal of Nursing 15(5) (2006), 9-22, 258262.

[25] Population Division, DESA. United Nations. World Population Ageing 2003, 1950-2050.

[26] M.J. Prince, The need for research on dementia in developing countries, Trop Med Health 2 (1997), 993-1000.

[27] The ICD-10 Classification of Mental and Behavioural Disor- ders: clinical descriptions and diagnostic guidelines, World Health Organization, Geneva, 1992.

[28] A. Wimo, B. Winblad, H. Aguero-Torres and E. von Strauss, The magnitude of dementia occurrence in the world, Alzheimer Dis Assoc Disord 17 (2004), 63-67.

[29] J. Woolham, Safe at Home, Hawker Publications Ltd, London, UK, 2006.

[30] World Health Organisation, World Health Report, Shaping the Future. Geneva, 2003. 